\title{
Novel HCN4 gene 'splice-site' mutation causing familial bradycardia
}

\author{
Beáta Csányi", Zoltán Hegedus², István $\mathrm{Nagy}^{2}$, Lidia Hategan², László Sághy², Miklós Csanády², \\ Tamás Forster ${ }^{2}$, Róbert Sepp ${ }^{2}$ \\ ${ }^{1}$ Second Department of Medicine and Cardiology Centre, University of Szeged, Szeged, Hungary \\ ${ }^{2}$ Biological Research Centre, Szeged, Hungary
}

The hyperpolarization-activated, cyclic nucleotide-gated ( $\mathrm{HCN})$ ion channel is the main pacemaker channel of the heart, which modulates the If mixed cationic current responsible for sinus node pacemaker potential. The major HCN channel of the heart is encoded by the HCN4 gene, mutations which were reported causing inherited sick sinus syndrome.

We performed genetic analysis of a proband with familial bradycardia. The female proband was diagnosed having sick sinus syndrome since the age of 28 . She had episodes of dizziness, symptoms of exercise intolerance, but no definite syncope occurred. On resting ECG 40-46/min sinus bradycardia was registered, Holter monitoring showed sinus rhythm with an average frequency of $58 / \mathrm{min}$. Even during active hours $38-48 / \mathrm{min}$ sinus bradycardia was noted with the lowest heart rate of $30-33 /$ min during sleep. During stress tests, preserved chronotrop competence was detec-

Received: $16^{\text {th }}$ Apr 2014

*Address for correspondence: Second Department of Medicine and Cardiology Centre, University of Szeged, Korányi fasor 6, H-6720 Szeged, Hungary

Phone: +3670-6122871

E-mail: csabea88@gmail.com ted with heart rate rising up to 123-150/min. Echocardiography showed normal cardiac structure and function with telesystolic mitral valve prolapse. Many of the family members of the patient have a similar disease.

Genetic analysis was performed on DNA extracted from peripheral blood. The whole coding sequence and the exonintron boundaries of HCN4 gene were amplified by polymerase chain reaction, and were direct sequenced.

In the patient's sample, a G-T transition was detected at the first nucleotide of the 'splice site' boundary of exon 5 and intron 5 (c.IVS5+1 G>T). We assume that the consequence of the mutation is that intron 5 is not cleaved during the RNA maturation. As it contains no cryptic stop codon, it is transcribed and translated full length. As a result of the mutation, the mutant protein is 34 amino acids longer than the wild type protein.

To summerize, we identified a new mutation (c.IVS5+1 G>T) in the HCN4 gene causing familial bradycardia. The mutation has never been reported before, therefore it represents a 'novel' mutation for sick sinus syndrome.

KEYWORDS: familial bradycardia, sick sinus syndrome, genetic mutation, HCN4 gene.

CITATION: Cardiol Croat. 2014;9(5-6):243. 